\title{
Role of Nursing Management to Assess Disability Prevention and Elimination of Lymphatic Filariasis
}

\author{
Gehad T. El Sherbini ${ }^{1 *}$, Nancy Osman Hany Kamel $^{1}$ and Morsy R. Geneedy ${ }^{2}$ \\ ${ }^{1}$ Department of Parasitology, Faculty of Medicine, Egypt \\ ${ }^{2}$ Department of Parasitology, Faculty of Medicine (Boys), Al Azhar University, Egypt \\ *Corresponding author
}

\section{A B S T R A C T}

Hygiene and skin care are effective and important interventions in the management of lymph edema secondary to lymphatic filariasis. Lymphatic filariasis has been identified as

Keywords

Lymphatic

filariasis,

Lymphoedema,

Skin care, Nursing

management

Article Info

Accepted:

14 December 2017

Available Online:

10 January 2018 one of the six diseases that can be potentially eliminated. We analyzed the impact on the quality of life that education and introduction of a designated nurse had on lymphoedema patients in a community that was endemic for lymphatic filariasis. Global programme to eliminate lymphatic filariasis has been launched, applying principal strategies of mass drug administration to interrupt transmission and morbidity management to prevent disability. filariasis. Patients' life quality was assessed using a Dermatology Life Quality Index Questionnaire (DLQI). At the same time they received education on appropriate hygiene, skin care techniques and simple exercises that encourage lymph drainage. A designated nurse was provided with educational materials and treatments. The DLQI life quality measure was repeated one year later. The DLQI improved for all patients and reported acute attacks were reduced. A paired t-test showed the improvement in DLQI to be highly significant $(\mathrm{P}=<0.0001)$. It is concluded that A nurse-led service combined with patient education in communities endemic for lymphatic filariasis is an effective intervention in improving the quality of life of patients with lymphoedema.

\section{Introduction}

Lymphatic Filariasis (LF) is an important public health problem in many tropical and sub-tropical countries. LF caused by Wuchereria bancrofti which is a mosquitotransmitted parasitic disease. The parasite have basically life cycles in man- adult worms living in lymphatic vessels whilst their offspring, the microfilariae circulate in the peripheral blood and are available to infect mosquito vectors when they come to feed. Filariasis is transmitted by the bite of infected vector mosquitoes. The parasite is deposited near the site of puncture. It passes through the punctured skin or may penetrate the skin on its own and finally reaches the lymphatic system. Climate is an important factor in the epidemiology of filariasis. It influences the breeding of mosquitoes, their longevity and also determines the development of parasite in the insect vector. Lymphatic filariasis is also associated with bad drainage. Inadequate sewage disposal and lack of town planning have aggravated the problem of filariasis in Egypt. The time interval between inoculation 
of infective larvae and the first appearance of detectable Mf is known as "pre patent period". The time interval from invasion of infective larvae to the development of clinical manifestation is known as the "Clinical incubation period". This period most commonly is 8 to 16 months. Clinical symptoms are Recurrent fever with chills, Pain in various organs, swelling with redness in lymph's, urticaria, erythematous rashes, conjunctivitis, pleurisy, enlarged tender lymph nodes, and palpable lymphatic vessels. Treatments are Suramin effective against adult roundworms Diethylcarbamazine (DEC) treatment for $W$. bancrofti, Albendazole and Flubendazole eliminate roundworms easily. Globally, 1.3 billion people are at risk of infection and about 120 million people are affected in 83 countries (Dreyer et al., 2000) and 1.3 billion persons are at risk of infection (Ottesen, 2006). (LF) leads to permanent lymphatic dysfunction in virtually all infected individuals and clinical disease in a subset of these (Dreyer et al., 2000).

A major sequalae of infection is lymphoedema of the limbs whereby lymphatic dysfunction can lead to swelling of the limbs which becomes permanent and, with repeated painful episodes of bacterial lymphangitis, known as (acute attacks), causes considerable acute morbidity and progression of lymphoedema to elephantiasis (Pani et al., 1995). lymphoedema has a negative impact on patient quality of life, however most studies on the impact on quality of life have been in the developed world on post- mastectomy patients (Woods, 1993; Launois, 2001).

The Dermatology Life Quality Index (DLQI) is a questionnaire that has been shown to be an accurate way of assessing the effect skin disease has on quality of life (Finlay and Khan, 1994). The DLQI contains ten questions which assess how skin disease has been affecting the individual's quality of life over the past week and covers symptoms, feelings, daily activities, leisure, work, personal relationships and treatment. Each question can score $0-3$ points with the total range being 0 30. As a measure of life quality the DLQI has been validated in the United Kingdom and applied to many skin conditions worldwide including in developing countries (Etemesi, 2002; Khilji et al., 2002). Furthermore, the DLQI has recently been validated as a tool to monitor changes in quality of life (Seim et al., 1999).

There has not yet been any published work on its use with lymphoedema patients. This study introduces the use of the DLQI for patients with limb lymphoedema. It was used in this setting, rather than a general health inventory to measure quality of life, as this study was looking in particular at the impact of the introduction of a hygiene and skin care regimen to patients. We analyzed the impact on the quality of life that education and introduction of a designated nurse had on lymphoedema patients in a community that was endemic for lymphatic filariasis, and this paper reports on the effect on the quality of life of lymphoedema patients following introduction of a hygiene and skin care regimen in one community endemic for lymphatic filariasis in Egypt.

\section{Materials and Methods}

Sahragt is a small village within the MitGhamer city Dakahlia governorate Egypt (Fig.1). Discussions with a local doctor during the first visit to implement mapping studies revealed patients with lymphoedema within the community who were currently receiving no organized care. The doctor identified a group of 15 patients known to be suffering from lymphoedema within his community served by a small local hospital (3 wards, daily medical out-patients). The principal researcher arranged to see these patients in the 
local hospital with a local nurse selected by the doctor. Patients were examined and the diagnosis of lymphoedema was confirmed clinically and classified using the scheme developed by Dreyer et al., in Brazil (Dreyer, 2002). All patients were then interviewed as to their disease history and current acute attack rates. They also were asked questions on current knowledge, attitudes and practices (KAP) and completed a Dermatology Life Quality Index (DLQI) questionnaire either on their own or with the assistance of the nurse if they were illiterate (2 patients). After the patients had been clinically assessed and completed the DLQI questionnaire, they were individually educated on LF and given appropriate advice on how to manage their lymphoedema, and educated on the importance of hygiene, skin care and elevation, as well as simple exercises to encourage lymph drainage. Treatment was given from a formulary of antibiotics (Penicillin) if current acute attack, antiseptics (potassium permanganate $2 \%$ ). Each patient was given a tube of topical antibacterial and anti-fungal cream if clinically indicated and a patient education leaflet (Dreyer et al., 1999). It was agreed that the nurse could offer advice and support to patients as and when they needed. Furthermore, the nurse could also provide education for any further patients who presented with lymphoedema. Diagnosis of filarial infection depended on the direct demonstration of the parasite always microfilariae in blood using relatively cumbersome techniques and having to take into account the periodicity (nocturnal or diurnal) of microfilariae. Alternative methods based on detection of antibodies by immunodiagnostic tests did not prove satisfactory since they both failed to distinguish between active and past infections and had problems with specificity owing to their cross-reactivity with common gastrointestinal parasites and other organisms (Sundarlal et al., 2014) (Table 1).

\section{Mass blood survey}

\section{The thick film}

The thick film made from capillary blood is still the most commonly used method for epidemiological assessment.20cu mm of blood is collected by a deep finger prick between $8.30 \mathrm{pm}$ and 12 midnight. A thick smear is prepared on a glass slide and the slide is dried and serially numbered. On the next day the blood films are dehaemoglobinished, stained, dried and examined for Mf under low power (Sunitapatney, 2005).

\section{DEC provocation test}

Mf can be induced to appear in blood in the day time by administering diethylcarbamazine (DEC) $100 \mathrm{mg}$ orally. Mf begins to reach their peak within 15 minutes and begin to decrease 2 hours later. The blood may be examined one hour after administration of DEC (Nainabhardwaj, 2014). Morbidity reduction measures, which emphasized the importance of hygiene and skin care were also explained and demonstrated.

The nurse was given further educational material and a supply of antiseptics, antibiotics and topical antibiotic and antifungal creams. Patients were advised to visit the nurse in the hospital outpatients if they had acute attacks or needed help with any issues. The nurse was invited to contact the researcher if she had any questions. Six months after the initial clinic study, the nurse attended a LF workshop for health care workers. At this point the nurse was provided with a patient video; 'Lymphatic Filariasis, over the course of the year, more patients attended the group. The clinic was visited approximately one year later and the patients were reassessed. The number of acute attacks during the last year was recorded as reported by the patient and the DLQI was repeated. 
Strategies used to management of lymphoedema to reduce the progression of the disease

\section{Looking after the skin}

This is an absolutely fundamental part of lymphoedema management in LF endemic parts of the world. These messages are being taught to those who are healthy as a preventive measure and to those with lymphoedema and skin changes in order to prevent progression of the disease to the more serious stages.

\section{Skin care messages}

Various skin care include:

Wash the skin carefully on a daily basis using soap and clean water, always rinse the skin carefully.

Dry the skin thoroughly but gently with a clean towel or soft cloth.

Apply an emollient to the skin if the skin is cracked or dry.

Check between toes and in skin folds for breaks in the skin and fungal infections treat with an appropriate antifungal or antibacterial product.

Wear comfortable shoes to protect the skin from traumatic damage.

\section{Problem meets us}

While these steps seem relatively simple to follow, in resource poor environments many issues remain problematic. For example, clean water may not be available and, even if it is, it may not be plentiful, soap may be unavailable (and/or of a very poor quality), an individual may not own a towel or even a piece of clean cloth to dry with, and emollients are often well beyond the resources of those who need them. Despite these potential problems, it appears that these simple skin hygiene measures can make a difference in terms of reducing the frequency of acute attacks (Kerketta et al., 2005) (Table 2).

\section{Elevation}

Elevation is most effective at reducing swelling in the early stages of the disease. Low level swelling may be totally reversed overnight if the leg is elevated. Ideally, the limb should be raised to the same height as the hip, but the individual must feel comfortable. The knee should be slightly flexed and pressure on the ankle/heel should be avoided (Dreyer et al., 2002). In communities where furniture is scarce, elevation may be achieved by rolling a piece of cloth to place under a sleeping mat or by using a box padded by a piece of cloth.

\section{Movement}

Movement and simple exercises that stimulate lymphatic flow were taught to the patients to manage lymphoedema. Vigorous running, aggravate lymphoedema. Therefore, movement should be gentle yet deliberate and carried out on a regular daily basis. While movement is focused on the foot, for example, circling and flexing it, movement of the whole limb is beneficial. Walking and foot exercises are probably the most effective ways of promoting lymph flow (Vaqas and Ryan, 2003). Bandages not used due to its costly and difficult to use effectively in poor environments, which may be dirty and hot with poor water supplies

\section{Results and Discussion}

Fifteen patients were initially seen who had a clinical diagnosis of lymphoedema and who completed a DLQI. Eleven patients were seen 
approximately one year later at the follow-up visit and completed the DLQI.4 of the initial patients were not seen on this visit as they had moved from the area, and were therefore excluded from the study (Follow up $=79 \%$ ). In addition, 5 patients were seen on the follow-up visit that had begun to attend the clinic over the past year. Of the 11 initial patients included in the study, 2 were female and 9 were male. Their ages ranged from $25-$ 65 . The KAP study showed that before the intervention. patients had received haphazard care, including use of local remedies and care from pharmacies, private doctors and hospital clinics for acute attacks. There was very limited. Skin care and none of the patients were aware of the importance of hygiene. The initial DLQI showed only a weak relationship to lymphoedema severity as assessed by Dreyer stage of the worst affected limb $(0.33$ Pearson's correlation coefficient). Obviously this is only one assessment of disease severity and factors such as frequency of acute attacks could also be taken into consideration in future analysis. The follow-up DLQI scores were the same or better for all 11 patients who had the two assessments. These DLQI scores ranged between $0-11$ points (mean score $=$ 4.1points) and the improvement varied between 0-15 points (mean improvement 6.8 points). A paired t-test (to assess the significance of the change in DLQI values for each person) gave a value of 5.9 with 10 degrees of freedom ( $\mathrm{p}=<0.0001$ ) (Fig. 3).

Table.1 Clinical picture of patients

\begin{tabular}{l|l}
\hline PICTURE & PATIENT PICTURE \\
\hline Recurrent fever with chills & Present \\
Pain in various organs & Present \\
Swelling with redness in lymph & Present \\
Urticaria & Not Present \\
Erythematous rashes & Present \\
Conjunctivitis & Present \\
Pleurisy & Not Present \\
Enlarged tender lymph nodes & Present \\
Palpalle lymphatic vessels & Present
\end{tabular}

Table.2 Problems meeting our work

\begin{tabular}{|l|c|c|}
\hline Reasons & No. Out of systematic & non-compliers \\
\hline 1 Fear of side reaction & $\mathbf{3 3}$ & $\mathbf{1 0 . 4}$ \\
\hline 2 No disease, why take & $\mathbf{1 1}$ & $\mathbf{3 . 5}$ \\
\hline medicine & $\mathbf{6}$ & $\mathbf{1 . 9}$ \\
3 Out of station & $\mathbf{6}$ & $\mathbf{1 . 9}$ \\
4 Current chronic disease & $\mathbf{4}$ & $\mathbf{1 . 3}$ \\
5 No faith in the tablet & $\mathbf{4}$ & $\mathbf{1 . 3}$ \\
6 Not received tablet & $\mathbf{3}$ & $\mathbf{0 . 9}$ \\
7 Do not like taking medicine & $\mathbf{2}$ & $\mathbf{0 . 6}$ \\
8 Not aware of the & $\mathbf{0 . 3}$ \\
programme & $\mathbf{6 6}$ & $\mathbf{2 0 . 8}$ \\
Total & &
\end{tabular}


Table.3 Perceptions among management used

\begin{tabular}{|c|c|c|c|}
\hline method & Most useful & Least useful & Problems \\
\hline $\begin{array}{l}\text { Management } \\
\text { distribution }\end{array}$ & $\begin{array}{l}\text { House to house } \\
\text { School } \\
\text { Work place }\end{array}$ & $\begin{array}{l}\text { Central guesthouse } \\
\text { within a village } \\
\text { Booths in public } \\
\text { places }\end{array}$ & $\begin{array}{l}\text { Large geographical area per team } \\
\text { Feral dogs } \\
\text { Loss of motivation } \\
\text { Working overtime with no } \\
\text { compensation } \\
\text { Distributing after dark } \\
\text { Inclement weather } \\
\text { Lack of transportation } \\
\text { Lack of community awareness } \\
\text { Rumors of side effects } \\
\text { Anxiety of taking many pills for one }\end{array}$ \\
\hline $\begin{array}{l}\text { Social } \\
\text { mobilization }\end{array}$ & $\begin{array}{l}\text { Visual aids showing disease } \\
\text { TV sessions } \\
\text { Outreach lectures }\end{array}$ & $\begin{array}{l}\text { T-shirts } \\
\text { Print materials }\end{array}$ & dose \\
\hline
\end{tabular}

Fig.1 Mosquito breeding ground (Sahragt Village)

Fig.2 Filaria clinic

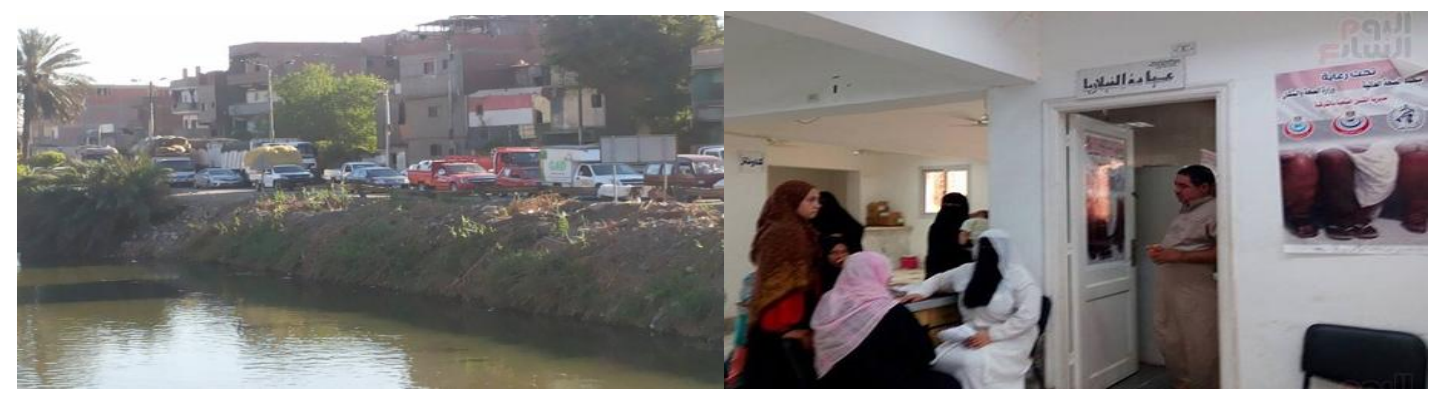

Fig.3 Flariasis cases

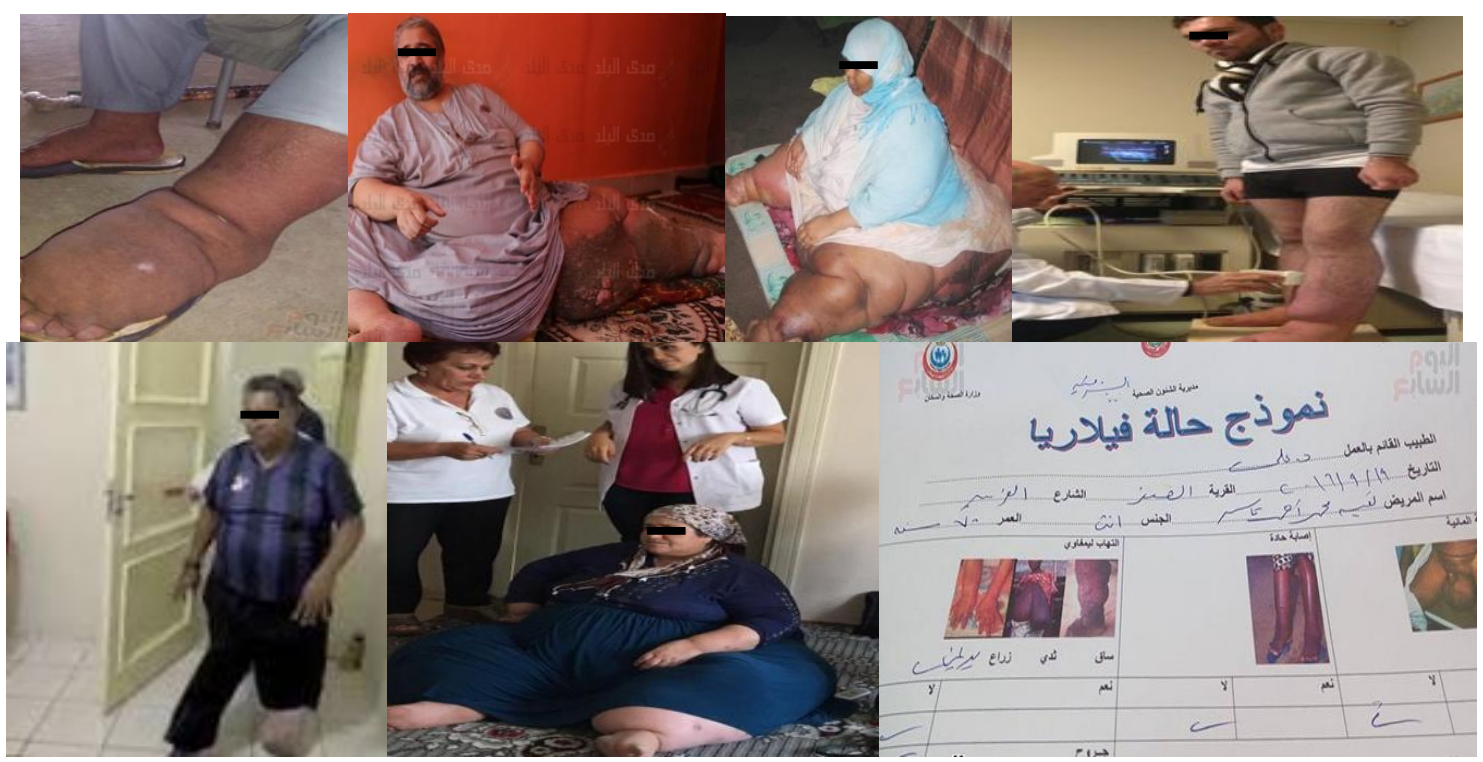


Fig.4 Filariasis before and after management
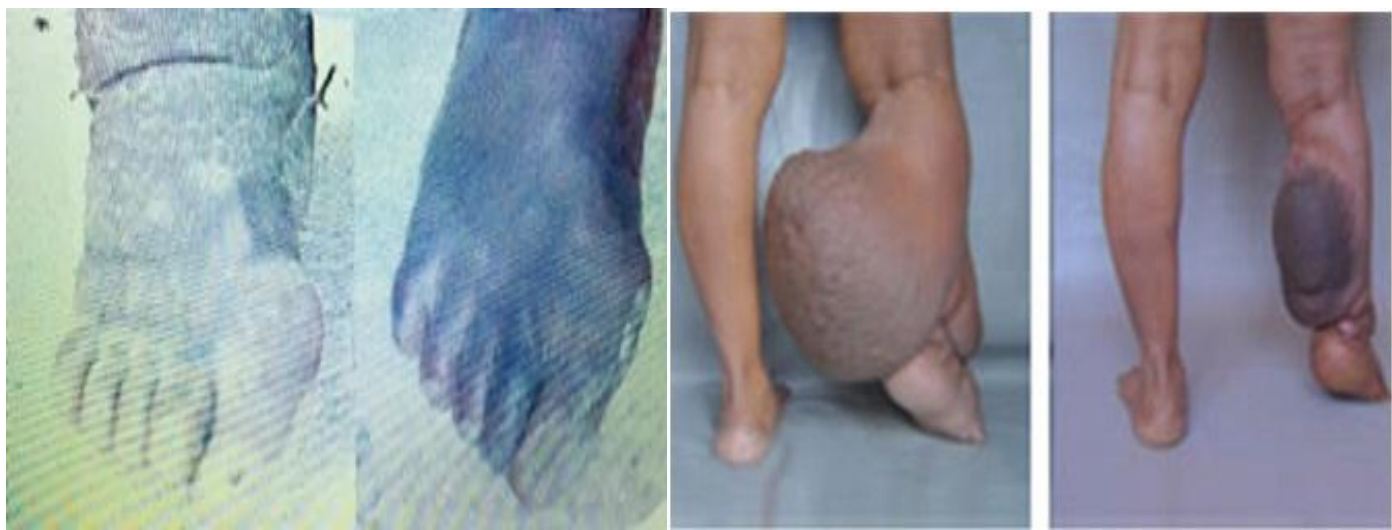

Patients also reported both a decrease in lymphoedema severity and notably in acute attack rates. In the initial visit, 10 out of the 11 patients had reported an acute attack in the preceding 6 months; however, no reports of acute attacks were made by any of the 11 patients in the 6 months preceding the follow up visit. It was not felt that other clinical outcomes could be analysed accurately in this small sample. The Dreyer stage did not change in any of the patients and limb size had not been reliably measured on the first visit but even though results were not measured objectively, patients did perceive subjective clinical improvement (Fig. 4).

This study shows that in this community, with only a very limited input of time and financial resources, a change/introduction in treatment regime can have a beneficial impact on patients with lymphoedema within a year. This intervention used the existing services within the community and gave them the necessary information enabling them to put into place more informed treatment. The intervention was easy to implement and required very limited follow up. This is valuable investment for a chronic condition, which is permanent once established. However, follow up is essential to see if the effect continues over time. It was felt from this preliminary experience that the DLQI was an appropriate tool to assess how limb lymphoedema affected patient quality of life and in particular to monitor an intervention that concentrated on skin care treatment. A study involving larger numbers of patients could assess in greater detail which features of life quality relate specifically to lymphoedema patients, and how this tool can be used alongside other life quality measures. There are a number of reasons why this intervention could have improved quality of life (as measured by the DLQI). In discussion with the patients and the nurse, likely contributing factors raised were reduced clinical severity (in particular a reduction in acute attacks) leading to an increased ability to work and perform daily functions. Other factors which were stated included the empowerment associated with an understanding of the condition and the ability to manage it individually. Also cited as important were the support from the clinic, the nomination of a clear focal point (the designated nurse) in the hospital, the ability to meet with other patients and the networks that were formed in the community. Interestingly, the DLQI scores in the 5 new patients were not as high as the initial group. This may reflect the fact that the improvements in the quality of service, as well as the establishment of the patient support group, benefited new patients as well. As this study was designed to provide the entire community with access to treatment there were no controls and therefore 
could be no estimation of the extent of a placebo effect. Further work on larger samples and more in-depth investigations of the degree, duration and cost of alternative approaches are needed to identify which of these factors had the greatest effect. The nurse was non-specialist but gained confidence from her work in this area. She felt extremely positive about the effect she was able to have on patients and the autonomy she felt. She only supplies of topical antibacterial and antifungal creams and did not have any clinical questions that she felt could not be answered by the educational material she was provided with. She has been able to educate other health workers, which raises the probability of sustainability of the intervention (Table 3).

Nurses have a significant role to play and the lymphoedema and dermatology specialists should enhance their impact by working together. A minimal amount of intervention in this small sample population has resulted in an improvement in patient quality of life and ability to function within their community. This study shows that low cost interventions, which provide an initial centre for patient treatment and education on disease management, can have a significant beneficial impact on outcomes.

\section{Goals for future}

According to medical experts the worldwide efforts to eliminate lymphatic filariasis is on track to potentially be successful by 2020 . A vaccine to be developed in the near future to finish this disease and to save as much people as we can from this disease.

\section{List of abbreviations used}

DLQI: Dermatology Life Quality Index

KAPB: Knowledge Attitudes Practices and Behavior

\section{References}

Dreyer G Assessment of Chronic Lymphoedema. In: Basic Lymphoedema Management Hollis Publishing 2002, 13-21.

Dreyer G, Addis D, Aguiar A, Bettinger J, Dreyer P, Luiz A, Miguel S, Neves M and Peterson A New hope for people with lymphoedema. Produced by: $N G O$ Amaury Coutinho and Division of Parasitic Diseases CDC Atlanta 1999, 1-16.

Dreyer G, Addiss D, Dreyer P, Noroes J 2002. Basic Lymphoedema Management: Treatment and Prevention of Problems Associated with Lymphatic Filariasis. Hollis Publishing Company, New Hampshire.

Dreyer G, Noroes J, Figueredo-Silva J and Piessens WF Pathogenesis of lymphatic disease in bancroftian filariasis: A clinical perspective. Parasitol Today 2000, 6:544-548.

Etemesi BA Quality of life in Tanzanian adults with chronic skin disease. Ann Dermatol Venereol 2002, 129:253.

Finlay AY and Khan GK Dermatology Life Quality Index (DLQI): A simple practical measure for routine clinical use. Clin Exp Dermatol 1994, 19:210216.

Kerketta A, Babu B, Rath K, Jangid P, Nayak A, Kar S 2005. A randomized clinical trial to compare the efficacy of three treatment regimens along with footcare in the morbidity management of filarial lymphoedema. Trop Med Int Health 10(7): 698-705.

Khilji FA, Gonzalez $M$ and Finlay AY Clinical meaning of change in Dermatology Life Quality Index scores. Br J Dermatol 2002, 147(Suppl 62):50.

Launois R, Mégnigbêto AC, Le Lay K and Alliot F Specific Quality of Life Scale in Upper Limb Lymphoedema Quality 
of Life Research Journal/Book/Report 2001, 10(3):291.

Nainabhardwaj,"Community health nursing", $2^{\text {nd }}$ edition, Jaypee publication, 2014. p. 85- 88

Ottesen, E.A., 2006. Lymphatic filariasis: treatment, control and elimination. Adv.Parasitol. 61, 395-441.

Pani SP, Yuvaraj J, Vanamaii P, Dhanda V, Michael E, Grenfeli B and Bundy D Episodic adenolymphangitis and lymphoedema in patients with bancroftian filariasis. Trans $R$ Soc Trop Med Hyg 1995, 89:72-74.

Seim AR, Dreyer G and Addis D Controlling morbidity and interrupting transmission: Twin pillars of lymphatic filariasis elimination. Rev Soc Bras Med Trop 1999, 32(3):325-328.

Shenoy RK, Sandhya K, Suma TK and
Kumaraswami V Prevention of acute adenolymphangitis in filariasis: Comparison of the efficacy of vermectin and diethylcarbamazine, each combined with local treatment of the affected limb. Ann Trop Med Parasitol 1998, 92:587-594.

Sundarlal, Adarsh, Pankaj, Textbook of community medicine, $4^{\text {th }}$ edition, CBS Publishers, 2014. p. 476- 477.

Sunitapatney,"Textbook of community health nursing, 1st edition, CBS Publishers, 2005. p. 327- 330.

Vaqas B and Ryan T 2003. Lymphoedema: pathophysiology and management in resource poor setting - relevance for LF control programmes. Filaria $J$ 2: 4.

Woods M Patients perceptions of breast cancer related lymphoedema. Eur $J$ Cancer Care (Engl) 1993, 2: 125-128.

\section{How to cite this article:}

Gehad, T. El Sherbini, Nancy Osman Hany Kamel and Morsy R. Geneedy. 2018. Role of Nursing Management to Assess Disability Prevention and Elimination of Lymphatic Filariasis. Int.J.Curr.Microbiol.App.Sci. 7(01): 1658-1666. doi: https://doi.org/10.20546/ijcmas.2018.701.201 